\title{
Median nerve behavior at different wrist positions among older male
}

Ping Yeap Loh, Hiroki Nakashima, Satoshi Muraki

The effect of wrist flexion-extension on the median nerve appearance, namely the crosssectional area (MNCSA) and the longitudinal (D1) and vertical (D2) diameters, was investigated among older adults $(\mathrm{N}=34)$. Ultrasound examination was conducted to examine the median nerve at different wrist angles (neutral; and $15^{\circ}, 30^{\circ}$, and $45^{\circ}$ extension and flexion), in both the dominant and nondominant hand. Median nerve behavior were significantly associated with wrist angle changes. The MNCSA at wrist flexion and extension were significantly smaller $(P<.001)$ compared with the neutral position in both the dominant and nondominant hand. The D1 and D2 were significantly reduced at flexion $(P<.001)$ and extension $(P<.001)$, respectively, in both the dominant and nondominant hand. Our results suggest that a larger flexion-extension angle causes higher compression stress on the median nerve, leading to increased deformation of the MNCSA, D1, and D2 among older adults. 
3 *Ping Yeap $\mathrm{LOH}^{1}$, Hiroki NAKASHIMA ${ }^{1}$, Satoshi MURAKI²

4

$5{ }^{1}$ Department of Human Science, Graduate School of Design, Kyushu University, Minami-ku, Fukuoka, 6 Japan

$7 \quad{ }^{2}$ Department of Human Science, Faculty of Design, Kyushu University, Minami-ku, Fukuoka, Japan 8

$9 *$ Corresponding Author

10 Name: Loh PY

11 E-mail address: lohpingyeap@gmail.com

12 Contact number: $+81-80-3958-9797$ 
Introduction

14 Carpal tunnel syndrome (CTS) is one of the most common peripheral entrapment neuropathies. CTS

15 causes peripheral nervous system changes with respect to morphological and functional characteristics

16 such as a reduction in sensory nerve conduction velocity (Mold et al., 2004; Verdu et al., 2005). Both

17 the motor and sensory symptoms of CTS have substantial effects on work performance and quality of

18 life. Indeed, the health-related quality of life is reduced in CTS patients compared with the general

19 population (Mold et al., 2004).

21 The United States Bureau of Labor Statistics (2008) estimated a 36.5\% increase of workers who are aged 22 55-64 years from 2006 to 2016. Notably, the incidence of CTS is highest in the age groups of 50-54 23 years and 75-84 years (Bland \& Rudolfer, 2003). The onset of CTS among older adults causes severe 24 symptoms such as weakness and atrophy of the thenar muscles, pain and numbness over the palm and 25 fingertips, as well as motor and sensory axon loss (Blumenthal, Herskovitz \& Verghese, 2006). CTS has 26 severe social and economic impacts such as a reduction in personal and industrial productivity, lower 27 income among employees, and increase of medical treatment costs (Foley, Silverstein \& Polissar, 2007).

28 Therefore, it is important to create an age-friendly working environment to reduce the risk of CTS among 29 the aging workforce.

31 The carpal tunnel volume becomes smaller as the wrist position changes into flexion or extension (Mogk $32 \&$ Keir, 2009). Changes of the wrist posture also cause deformation of the median nerve at the carpal 33 tunnel. Wrist flexion-extension positions cause the median nerve cross-sectional area (MNCSA) to 34 become smaller, whereas wrist radial-ulnar deviations do not have significant effects on the MNCSA 35 (Loh \& Muraki, 2014). Loh and Muraki (2015) reported that a higher wrist flexion/extension position 36 causes increased deformation of the MNCSA compared with the neutral wrist position. 
38 The aging process is known to cause physiological and anatomical changes to the hands as well as the 39 peripheral nervous system (Carmeli, Patish \& Coleman, 2003). Loh and Muraki (2015) reported changes

40 in median nerve behavior at different wrist angles among young participants. However, the median nerve

41 behaviors with respect to changes in the wrist angle among older adults are not well understood.

42 Therefore, the objective of the present study was to investigate the age-related changes of median nerve 43 behavior, namely MNCSA and the longitudinal (D1) and vertical (D2) diameters, at different wrist angles 44 among older adults.

\section{Materials and Methods}

\section{Participants}

48 This study was approved by the Ethics Committee of the Faculty of Design at Kyushu University

49 (Approval number, 141; June 04, 2013). In previous study (Loh \& Muraki, 2015), there was no difference 50 in deformation pattern in the MNCSA, D1, and D2 between the young female and male groups. 51 Therefore, in this study, we recruited only male participants. Thirty-four healthy men were recruited and 52 written informed consent was obtained (Table 1). The inclusion criteria for participation were that the 53 subject was able and willing to provide informed consent and was above 60 years of age. The exclusion 54 criteria for participation were patients with CTS, diabetes, or a history of wrist surgery or wrist fracture. 55 All participants were free of signs and symptoms of CTS, as indicated by screening tests, including the 56 Boston Carpal Tunnel Questionnaire, Phalen Test, and CTS Tinel Test (LaJoie et al., 2005; Sambandam 57 et al., 2008). The Edinburgh Handedness Inventory (Oldfield, 1971) was used to identify the handedness 58 of the participants. Table 1 presents the demographic data of the participants. 


\section{Ultrasound Examination}

61 The ultrasound examination protocol was conducted as described by Loh and Muraki (2015). Ultrasound 62 examination was conducted with the LOGIQ e ultrasound system (GE Healthcare; USA) and a 7.0-mmthick sonar pad (Nippon BXI Inc.; Tokyo, Japan) as a coupling medium. A total of seven wrist positions were examined as follows: neutral $\left(0^{\circ}\right) ; 15^{\circ}, 30^{\circ}$, and $45^{\circ}$ extension; and $15^{\circ}, 30^{\circ}$, and $45^{\circ}$ flexion. A wrist goniometer was used to determine the passive wrist angle during the ultrasound examination. The fulcrum of the goniometer was placed at the triquetrum, while the static arm was placed parallel to the ulnar bone and the moveable arm was placed parallel to the fifth metacarpal bone. Three images were taken for each wrist position during the ultrasound examination. Ultrasound examination with the passive wrist angle was repeated for both the dominant and nondominant hand. 西

The examiner palpated the pisiform bony mark and placed the ultrasound probe at the pisiform level to identify the proximal edge of the transverse carpal ligament and the median nerve in the transverse plane by a hypoechogenic rim, which contained hypoechogenic nerve fascicles. The MNCSA, D1, and D2 (Fig. 1) were quantified using ImageJ software (Schneider, Rasband \& Eliceiri, 2012). The MNCSA was measured by the tracing method along the hypoechogenic rim of the median nerve; the D1 and D2 were measured as described by Duncan et al. (1999). Loh and Muraki (2015) reported good to excellent interand intrarater reliability in quantification of the MNCSA and median nerve diameters. Mean values of three images were calculated to represent the MNCSA, D1, and D2 at each wrist angle.

\section{Deformation Percentage}

1 Deformation percentages of the MNCSA, D1, and D2 at different wrist angles relative to values in the neutral wrist position were calculated with the following equation: 


$$
\text { Deformation Percentage }=\frac{\text { Neutral wrist }- \text { Different wrist angle }}{\text { Neutral wrist }} \times 100 \%
$$

\section{Statistical Analysis}

85 Statistical analysis was performed using SPSS version 21.0 software (IBM Corporation; Chicago, IL, 86 USA). The sample characteristics of MNCSA in both the dominant and nondominant hand were 87 examined by the Shapiro-Wilk’s normality test.

88

89 The paired $t$-test was used to analyze differences in MNCSA, D1, and D2 between the dominant and 90 nondominant hands at neutral position. Two-way repeated analysis of variance $(2 \times 7$ factorial design $)$ 91 was conducted with wrist side (dominant and nondominant) and the seven wrist angles (neutral $\left[0^{\circ}\right] ; 15^{\circ}$, $9230^{\circ}$, and $45^{\circ}$ extension; and $15^{\circ}, 30^{\circ}$, and $45^{\circ}$ flexion) as factors to examine differences in MNCSA, D1, 93 and D2 at different wrist positions. Greenhouse-Geisser correction was used in the analysis of variance

94 because the assumption of sphericity was violated, as indicated by Mauchly's test. Post-hoc pairwise 95 Bonferroni-corrected comparison was used to examine mean differences in the factors. The significance 96 level was set at $0.05(5 \%)$ for all statistical tests.

97

98 Results

99 Sample Characteristics

100 The Shapiro-Wilk's test $(\mathrm{P}>.05)$, and visual inspection of histograms, normal Q-Q plots, and box plots 101 indicated the MNCSA values of both hands were approximately normally distributed and slightly skewed 102 and kurtotic (Table 2) (Cramer \& Howitt, 2004; Doane \& Seward, 2011; Razali \& Wah, 2011; Shapiro $103 \&$ Wilk, 1965). 
105

106

107

108

109

110

111

112

113

114

115

116

117

118

119

120

121

122

123

124

125

Comparison of Median Nerve Behavior between the Dominant and Nondominant Hands at Neutral Wrist Position

The MNCSA and D1 values of the dominant hand were significantly larger $(\mathrm{P}<.001)$ than those of the nondominant hand, while D2 showed no significant difference between the hands $(\mathrm{P}=.137)($ Table 3$)$.

\section{Change in MNCSA at Different Wrist Angles}

No significant wrist angle $\times$ handedness interaction was found $(\mathrm{F}[4.1,135.9]=1.135, \mathrm{P}=.267)$. However, wrist angle had a significant effect on MNCSA, which became smaller when the wrist changed from a neutral to a flexion or extension position in both the dominant and nondominant hands. The MNCSA at neutral position $\left(0^{\circ}\right)$ was significantly larger as compared with $15^{\circ}, 30^{\circ}$, and $45^{\circ}$ flexion and extension, respectively (Fig. $2 \mathrm{a}$ and $2 \mathrm{~b}$ ). The mean values and the deformation percentages of the MNCSA at different wrist positions are presented in Tables 4 and 5, respectively.

(1)

\section{Changes in D1 and D2 at Different Wrist Angles}

The mean D1 and D2 values at different wrist angles are presented in Table 5. The results showed no significant wrist angle $\times$ handedness interaction for both D1 and D2 (D1: F [4.0, 130.5] =.399, P = .807; $\mathrm{D} 2: \mathrm{F}[3.6,118.0]=.601, \mathrm{P}=.644)$. Wrist flexion had a significant influence on $\mathrm{D} 1$, which became smaller compared with that at neutral position (Fig. 2c and 2d). Meanwhile, wrist extension caused a significant decrease in D2 (Fig. 2e and 2f). The mean values and deformation percentages of the D1 and D2 at different wrist positions are presented in Table 4 and Tables 6 and 7, respectively. 
126

127

128

129

130

131

132

133

134

135

136

137

138

139

140

141

142

143

144

145

146

147

148

\section{Discussion}

\section{Aging in the Peripheral Nerve}

The aging process causes specific changes to the peripheral nerve such as a reduction in conduction velocity, as well as biomechanical and morphological changes (Verdu et al., 2000). The process of peripheral nerve degeneration is associated with the phenomenon of the loss of nerve fibers (Kerasnoudis

et al., 2013). Furthermore, peripheral nerve morphological studies suggested that aging causes a reduction in the percentage of myelinated axons while increasing the epineural and fascicular areas, with a larger neural cross-sectional area observed in the elderly group (Kerasnoudis et al., 2013; Kundalić et al., 2014). The mean MNCSA value in young adults and the elderly group ( $>60$ years old) were 8.75 $\mathrm{mm}^{2}$ and $9.12 \mathrm{~mm}^{2}$, respectively (Kerasnoudis et al., 2013). The mean values of the MNCSA at the neutral wrist position in the older adults (Table 4) were larger compared with those of the young adults $\left(\right.$ Dominant $=8.36 \pm 1.47 \mathrm{~mm}^{2} ;$ Nondominant $\left.=7.32 \pm 1.40 \mathrm{~mm}^{2}\right)$ reported in our previous study $($ Loh $\&$ Muraki, 2015). Furthermore, the D1 values of the older adults were generally larger than those of the young adults, which was not the case for the D2 values. Therefore, the longer D1 may be the main factor contributing to the larger MNCSA observed among older adults.

\section{Deformation of the MNCSA among Older Adults}

A larger angle in wrist flexion and extension led to increased deformation of the MNCSA at the proximal carpal tunnel level (Table 5). The carpal tunnel volume decreases as the wrist moves to extension and flexion positions, which leads to increased intra-carpal tunnel pressure, resulting in median nerve compression from the surrounding tendons within the carpal tunnel (Mogk \& Keir, 2009; Vital et al., 1990). The CSA of a nerve is reduced when it undergoes elongation, in a process known as transverse contraction (Keir \& Rempel, 2005). The epineurium layer helps to constrain the inner core pressure of 
149 the nerve and contributes to the stiffness of the nerve in resisting the transverse contraction (Keir \& 150 Rempel, 2005; Millesi, Zoch \& Reihsner, 1995).

152 Morphometric studies of the peripheral nerve have indicated the quantitative and qualitative changes that 153 occur during the aging process, such as an increased CSA and thickening of the peripheral nerve sheath 154 of a peripheral nerve (Kundalić et al., 2014; Topp \& Boyd, 2006). The thicker epineurium in older adults, 155 which may explain their larger MNCSA, may affect the biomechanical properties of a nerve owing to 156 the stress imposed on the nerve during joint movement. Similar to the observations in young adults (Loh $157 \&$ Muraki, 2015), an increase of $15^{\circ}$ in wrist flexion or extension caused a significant reduction and 158 higher deformation percentage of the MNCSA (Fig. 2a and 2b). However, the deformation of MNCSA 159 at $45^{\circ}$ flexion and extension was reduced in older adults (-20\%) compared with young adults $(-25 \%){ }^{9}$

160 Therefore, the thicker peripheral nerve sheath among older adults may help to provide stronger inner 161 pressure of a nerve while responding to the stretch and compression stress occurring during wrist angle 162 changes.

\section{Deformation of the D1 and D2 among Older Adults}

165 At a neutral wrist position, the D1 values of older adults (Table 4) were larger than those of the young 166 group of our previous study (Loh \& Muraki, 2015) $($ Dominant $=5.09 \pm 0.59 \mathrm{~mm}$; Nondominant $=4.79$ $167 \pm 0.66 \mathrm{~mm}$ ), whereas the D2 values were similar between older adults (Table 4) and young adults 168 (Dominant $=2.11 \pm 0.23 \mathrm{~mm}$; Nondominant $=1.95 \pm 0.26 \mathrm{~mm})$. Furthermore, the deformation pattern 169 of D1 at wrist flexion and D2 at extension positions are similar to those of the young adults as reported 170 by Loh and Muraki (2015). The compression stress and elongation at wrist flexion-extension caused the 171 D1 to become smaller as the wrist position changed into flexion positions, whereas the $\mathrm{D} 2$ became shorter 
172 at the wrist extension positions (Fig. 2c-2f). The loose space within the epineural tube enables a change

173 of the fascicular position so that the peripheral nerve can adapt to the biomechanical stress such as strain,

174 excursion, and transverse contraction experienced during limb movement (Keir \& Rempel, 2005;

175 Sladjana, Ivan \& Bratislav, 2008). Therefore, the nerve behavior at different joint positions of older

176 adults can be observed via changes in the nerve diameters.

177

178 The carpal tunnel is a confined space and is tightly packed with flexor tendons and a median nerve. A

179 larger D1 and MNCSA may indicate that the median nerve occupies relatively more space at the carpal

180 tunnel. Therefore, the higher deformation percentages of D1 and D2 at different wrist positions among

181 older adults could reflect their larger MNCSA at the proximal carpal tunnel level. However, the lower

182 deformation observed among older adults (Fig. 3a and 3b) suggested age-related changes of median

183 nerve behavior at different wrist angles. The lower deformation percentages among older adults may

184 indicate a thickened median nerve sheath and lack of movement among the polyfascicular tube within

185 the epineural tube. Undulation mechanisms play an important role when a nerve is exposed to the

186 elongation and shortening stress across a joint or multijoint, because the loose arrangement of the

187 perineural tube within the epineurium sheath allows for volume adaptation of a nerve (Keir \& Rempel,

188 2005; Sladjana, Ivan \& Bratislav, 2008). When a joint is flexed, the nerve that spans across the joint

189 becomes shorter by increasing the number of perineurium undulations. In contrast, when the joint is

190 extended, the folded undulations will be straightened in response to the longitudinal elongation stress

191 (Keir \& Rempel, 2005). Therefore, undulation mechanisms may cause the nerve diameters to change

192 accordingly during joint movement. Furthermore, the physical connection between the core and sheath

193 of the nerve, and the total fascicle number within the nerve are known to affect the stiffness of a nerve

194 (Tillett et al., 2004). The increase in the ratio between the perineural thickness of the sural nerve and the 
195 fascicular diameter may increase the risk of chronic trauma over the nerve (Tohgi, Tsukagoshi \&

196 Toyokura, 1977). Therefore, aging nerves are more vulnerable to stress.

197

198 The aging process causes degenerative changes of wrist-carpal and carpal joints, which leads to

199 decreased mobility of wrist movements and affects older adults in their occupation and daily life 200 activities (Carmeli, Patish \& Coleman, 2003). The diameters of the carpal tunnel may increase with the 201 aging process, as the carpal tunnel volume of older adults are larger than those of younger adults (Pierre202 Jerome, Bekkelund \& Nordstrøm, 1997). Our results suggest that the MNCSA of the median nerve is 203 larger among older adults, with a longer D1, compared with younger adults. The median nerve undergoes 204 shear strain and compression stress owing to the excursion and displacement of tendons within the 205 confined space of the carpal tunnel (van Doesburg et al., 2010). The long-term effects of transverse 206 contraction force, shear strain, and compression stress within the narrow carpal tunnel may cause the 207 appearance of the median nerve to change with the aging process.

209 Aging Workforce and CTS

210 Age-related anatomical and physiological changes have strong correlations with decreased functions in 211 the central nervous system as well as the peripheral nervous system. Changes in the peripheral nervous 212 system among older adults affects the somatosensory system, physical work performance, and quality of 213 life (Carmeli, Patish \& Coleman, 2003; Sachs, 2008). Blumenthal et al. (2006) hypothesized that older 214 adults underreport their CTS symptoms, which results in severe median nerve entrapment compared with 215 young adults, as indicated in muscle wasting and nerve conduction studies. 
217 Humans have distinct hand functions such as a powerful grip, precision grip, and precise thumb and

218 finger movements. The normal aging process leads to a certain degree of deterioration in hand functions

219 such as a decrease in manual dexterity and loss of hand strength, which in turn affects the strength, motor

220 skills, work tolerance level, and speed of movement. In view of the increasing population of the aging

221 workforce and the implication of age-related problems of the upper extremities, specific ergonomics

222 considerations pertaining to the wrist angle in the design of tools and equipment and in the workplace

223 are required to minimize the compression stress on the median nerve.

224

225

226

227

228

229

230

231

232

233

234

235

236

237

238

239

240

\section{Acknowledgements}

The authors thank all of the individuals who participated in this study.

\section{References}

Bland JD, Rudolfer SM. 2003. Clinical surveillance of carpal tunnel syndrome in two areas of the United Kingdom, 1991-2001. Journal of neurology, neurosurgery, and psychiatry, 74:1674-1679.

Blumenthal S, Herskovitz S, Verghese J. 2006. Carpal tunnel syndrome in older adults. Muscle \& nerve, 34:78-83.

Bureau of Labor Statistics. 2008. Spotlight on statistics: Older workers. Washington, DC: U.S. Department of Labor.

Carmeli E, Patish H, Coleman R. 2003. The Aging Hand. The Journals of Gerontology Series A: Biological Sciences and Medical Sciences, 58:M146-M152.

Cramer D, Howitt D . 2004. The SAGE Dictionary of Statistics. SAGE Publications, Ltd.

Doane DP, Seward LE. 2011. Measuring Skewness: A Forgotten Statistic?. Journal of Statistics Education, 19.

Duncan I, Sullivan P, Lomas F. 1999. Sonography in the diagnosis of carpal tunnel syndrome. AJR.American journal of roentgenology, 173:681-684.

Foley M, Silverstein B, Polissar N. 2007. The economic burden of carpal tunnel syndrome: long-term earnings of CTS claimants in Washington State. American Journal of Industrial Medicine, 50:155-172. 
244 Keir PJ, Rempel DM. 2005. Pathomechanics of Peripheral Nerve Loading: Evidence in Carpal Tunnel 245 Syndrome. Journal of Hand Therapy, 18:259-269.

246

247

248

249

250

251

252

253

254

255

256

257

Kerasnoudis A, Klasing A, Behrendt V, Gold R, Yoon MS. 2013. Intra- and internerve cross-sectional area variability: new ultrasound measures. Muscle \& nerve, 47:146-147.

Kundalić B, Ugrenović S, Jovanović I, Stefanović N, Petrović V, Kundalić J, Pavlović M, Antić V. 2014.

Analysis of Fascicular Structure and Connective Tissue Sheaths in Sural Nerve during Aging. Acta Facultatis Medicae Naissensis, 31:113-120.

LaJoie AS, McCabe SJ, Thomas B, Edgell SE. 2005. Determining the sensitivity and specificity of common diagnostic tests for carpal tunnel syndrome using latent class analysis. Plastic and Reconstructive Surgery, 116:502-507.

Loh, P.Y., Muraki,S. 2014. Effect of Wrist Deviation on Median Nerve Cross-Sectional Area at Proximal Carpal Tunnel Level. Iranian Journal of Public Health, 43:180-185.

Millesi H, Zoch G, Reihsner R. 1995. Mechanical properties of peripheral nerves. Clinical orthopaedics and related research, (314):76-83.

Mogk JPM, Keir PJ. 2009. The effect of landmarks and bone motion on posture-related changes in carpal tunnel volume. Clinical Biomechanics, 24:708-715.

Mold JW, Vesely SK, Keyl BA, Schenk JB, Roberts M. 2004. The prevalence, predictors, and consequences of peripheral sensory neuropathy in older patients. The Journal of the American Board of Family Practice / American Board of Family Practice, 17:309-318.

Oldfield RC. 1971. The assessment and analysis of handedness: the Edinburgh inventory. Neuropsychologia, 9:97-113.

Pierre-Jerome C, Bekkelund SI, Nordstrlo m R. 1997. Quantitative MRI analysis of anatomic dimensions of the carpal tunnel in women. Surgical and radiologic anatomy : SRA, 19:31-34.

Razali N, Wah YB. 2011. Power comparisons of Shapiro-Wilk, Kolmogorov-Smirnov, Lilliefors and Anserson-Darling tests. Journal of Statistical Modeling and Analytics, 2:21-33.

Sachs GM. 2008. Peripheral neurology: special concerns in the elderly. Medicine and health, Rhode Island, 91:142-143.

Sambandam SN, Priyanka P, Gul A, Ilango B. 2008. Critical analysis of outcome measures used in the assessment of carpal tunnel syndrome. International orthopaedics, 32:497-504.

Schneider CA, Rasband WS, Eliceiri KW. 2012. NIH Image to ImageJ: 25 years of image analysis. Nature methods, 9:671-675. 
276 Shapiro SS, Wilk MB. 1965. An analysis of variance test for normality (complete samples). Biometrika, $277 \quad 52: 591-611$.

278 Sladjana UZ, Ivan JD, Bratislav SD. 2008. Microanatomical structure of the human sciatic nerve. 279 Surgical and Radiologic Anatomy, 30:619-626.

280 Tillett RL, Afoke A, Hall SM, Brown Ra, Phillips JB. 2004. Investigating mechanical behaviour at a 281 core-sheath interface in peripheral nerve. Journal of the peripheral nervous system : JPNS, 9:255-262.

282 Tohgi H, Tsukagoshi H, Toyokura Y. 1977. Quantitative changes with age in normal sural nerves. Acta 283 Neuropathologica, 38:213-220.

284 Topp KS, Boyd BS. 2006. Structure and biomechanics of peripheral nerves: nerve responses to physical 285 stresses and implications for physical therapist practice. Physical Therapy, 86:92-109.

286 Topp KS, Boyd BS. 2012. Peripheral Nerve: From the Microscopic Functional Unit of the Axon to the 287 Biomechanically Loaded Macroscopic Structure. Journal of Hand Therapy, 25:142-152.

288 van Doesburg MH, Yoshii Y, Villarraga HR, Henderson J, Cha SS, An KN, Amadio PC. 2010. Median 289 nerve deformation and displacement in the carpal tunnel during index finger and thumb motion. Journal 290 of orthopaedic research : official publication of the Orthopaedic Research Society, 28:1387-1390.

291 Verdu E, Ceballos D, Vilches JJ, Navarro X. 2000. Influence of aging on peripheral nerve function and 292 regeneration. Journal of the peripheral nervous system : JPNS, 5:191-208.

293 Vital A, Vital C, Rigal B, Decamps A, Emeriau JP, Galley P. 1990. Morphological study of the aging 294 human peripheral nerve. Clinical neuropathology, 9:10-15. 


\section{Table $\mathbf{1}$ (on next page) \\ Demographic data of participants $(n=34)$.}




\begin{tabular}{lcc}
\hline & & Mean \pm SD \\
\hline Age (years) & & $70.9 \pm 5.2$ \\
Height $(\mathrm{cm})$ & $164.9 \pm 6.1$ \\
Weight $(\mathrm{kg})$ & & $62.5 \pm 10.1$ \\
BMI $\left(\mathrm{kg} / \mathrm{m}^{2}\right)$ & Right & $22.9 \pm 2.9$ \\
Wrist Circumference $(\mathrm{mm})$ & Left & $167.3 \pm 9.7$ \\
& Right hand Dominant & $165.1 \pm 9.2$ \\
Handedness & Left hand Dominant & 33 \\
& & 1 \\
\hline
\end{tabular}

2 
Table 2 (on next page)

Normality test for median nerve cross-sectional area $(n=34)$.

$\mathrm{M}=$ mean; $\mathrm{SE}=$ standard error 


\begin{tabular}{lccc}
\hline Wrist & $\begin{array}{c}\text { Skewness } \\
(\mathrm{M} \pm \mathrm{SE})\end{array}$ & $\begin{array}{c}\text { Kurtosis } \\
(\mathrm{M} \pm \mathrm{SE})\end{array}$ & $\begin{array}{c}\text { Shapiro-Wilk Test } \\
\text { (P value) }\end{array}$ \\
\hline Dominant & $0.46 \pm 0.40$ & $-0.59 \pm 0.79$ & .233 \\
Nondominant & $0.52 \pm 0.40$ & $-0.42 \pm 0.79$ & .188 \\
\hline
\end{tabular}

2 


\section{Table 3(on next page)}

Comparison between dominant and nondominant hands at wrist neutral $(n=34)$.

MNCSA = median nerve cross-sectional area; $\mathrm{D} 1=$ median nerve longitudinal diameter; $\mathrm{D} 2=$ median nerve vertical diameter 


\begin{tabular}{lcccc}
\hline & Dominant hand & Nondominant hand & $\mathrm{t}$ & $\mathrm{P}$ \\
\hline MNCSA $\left(\mathrm{mm}^{2}\right)$ & $9.65 \pm 2.17$ & $8.70 \pm 1.88$ & 7.186 & $<.001$ \\
D1 $(\mathrm{mm})$ & $6.02 \pm 0.91$ & $5.58 \pm 0.80$ & 5.398 & $<.001$ \\
D2 $(\mathrm{mm})$ & $2.01 \pm 0.21$ & $1.96 \pm 0.25$ & 1.524 & .137 \\
\hline
\end{tabular}

2 


\section{Table 4(on next page)}

Mean values of median nerve cross-sectional area, longitudinal diameter and vertical diameter at different wrist positions.

MNCSA = median nerve cross-sectional area; D1 = median nerve longitudinal diameter; D2 = median nerve vertical diameter; Dom $=$ dominant hand; Nondom $=$ nondominant hand 


\begin{tabular}{|c|c|c|c|c|c|c|}
\hline \multirow{2}{*}{ Wrist Angle } & \multicolumn{2}{|l|}{$\begin{array}{c}\text { MNCSA } \\
\left(\mathrm{mm}^{2}\right)\end{array}$} & \multicolumn{2}{|l|}{ D1 (mm) } & \multicolumn{2}{|l|}{$\mathrm{D} 2(\mathrm{~mm})$} \\
\hline & Dom & Nondom & Dom & Nondom & Dom & Nondom \\
\hline Flexion $45^{\circ}$ & $7.65 \pm 1.79$ & $6.92 \pm 1.46$ & $4.78 \pm 1.04$ & $4.44 \pm 0.91$ & $2.03 \pm 0.24$ & $1.98 \pm 0.26$ \\
\hline Flexion $30^{\circ}$ & $8.08 \pm 2.04$ & $7.39 \pm 1.59$ & $5.09 \pm 1.08$ & $4.81 \pm 0.82$ & $2.02 \pm 0.26$ & $1.95 \pm 0.23$ \\
\hline Flexion $15^{\circ}$ & $8.92 \pm 2.09$ & $8.10 \pm 1.78$ & $5.55 \pm 1.07$ & $5.21 \pm 0.91$ & $2.00 \pm 0.20$ & $1.96 \pm 0.19$ \\
\hline $\operatorname{Neutral}\left(0^{\circ}\right)$ & $9.65 \pm 2.17$ & $8.70 \pm 1.88$ & $6.02 \pm 0.91$ & $5.58 \pm 0.80$ & $2.01 \pm 0.21$ & $1.96 \pm 0.25$ \\
\hline Extension $15^{\circ}$ & $8.87 \pm 1.95$ & $8.02 \pm 1.61$ & $5.95 \pm 0.74$ & $5.59 \pm 0.69$ & $1.88 \pm 0.23$ & $1.80 \pm 0.24$ \\
\hline Extension $30^{\circ}$ & $8.09 \pm 1.79$ & $7.43 \pm 1.53$ & $5.81 \pm 0.70$ & $5.49 \pm 0.74$ & $1.73 \pm 0.25$ & $1.72 \pm 0.26$ \\
\hline Extension $45^{\circ}$ & $7.52 \pm 1.62$ & $6.91 \pm 1.46$ & $5.80 \pm 0.73$ & $5.47 \pm 0.82$ & $1.63 \pm 0.23$ & $1.59 \pm 0.20$ \\
\hline
\end{tabular}


Table 5(on next page)

Deformation percentage of median nerve cross-sectional area (MNCSA) $\left(\mathrm{mm}^{2}\right)$ at difference wrist positions when compare to wrist neutral position.

Dom $=$ dominant hand; NonDom $=$ nondominant hand; Flex $=$ flexion; Extn $=$ extension; NA

$=$ Not applicable 


\begin{tabular}{lccccccc}
\hline $\begin{array}{l}\text { MNCSA } \\
\left(\mathrm{mm}^{2}\right)\end{array}$ & Flex & Flex & Flex & Neutral & $\begin{array}{c}\text { Extn } \\
15^{\circ}\end{array}$ & $\begin{array}{c}\text { Extn } \\
30^{\circ}\end{array}$ & $\begin{array}{c}\text { Extn } \\
45^{\circ}\end{array}$ \\
\hline Dom & $-20.3 \%$ & $-16.3 \%$ & $-8.4 \%$ & NA & $-7.8 \%$ & $-15.8 \%$ & $-21.6 \%$ \\
NonDom & $-19.8 \%$ & $-14.4 \%$ & $-7.8 \%$ & NA & $-7.4 \%$ & $-14.1 \%$ & $-20.0 \%$ \\
\hline
\end{tabular}


Table 6(on next page)

Deformation percentage of median nerve longitudinal diameter (D1) (mm) at difference wrist positions when compare to wrist neutral position.

Dom $=$ dominant hand; NonDom $=$ nondominant hand; Flex $=$ flexion; Extn $=$ extension; NA

$=$ Not applicable 


\begin{tabular}{|c|c|c|c|c|c|c|c|}
\hline \multirow{2}{*}{$\mathrm{D} 1$ (mm) } & Flex & Flex & Flex & \multirow{2}{*}{ Neutral } & Extn & Extn & Extn \\
\hline & $45^{\circ}$ & $30^{\circ}$ & $15^{\circ}$ & & $15^{\circ}$ & $30^{\circ}$ & $45^{\circ}$ \\
\hline Dom & $-20.6 \%$ & $-15.6 \%$ & $-9.8 \%$ & NA & $-0.7 \%$ & $-2.8 \%$ & $-3.0 \%$ \\
\hline NonDom & $-20.4 \%$ & $-13.6 \%$ & $-8.2 \%$ & NA & $0.7 \%$ & $-1.2 \%$ & $-1.3 \%$ \\
\hline
\end{tabular}


Table 7 (on next page)

Deformation percentage of median nerve vertical diameter (D2) (mm) at difference wrist positions when compare to wrist neutral position.

Dom $=$ dominant hand; NonDom $=$ nondominant hand; Flex $=$ flexion; Extn $=$ extension; NA

$=$ Not applicable 


\begin{tabular}{|c|c|c|c|c|c|c|c|}
\hline \multirow{2}{*}{$\mathrm{D} 2(\mathrm{~mm})$} & Flex & Flex & Flex & \multirow{2}{*}{ Neutral } & Extn & Extn & Extn \\
\hline & $45^{\circ}$ & $30^{\circ}$ & $15^{\circ}$ & & $15^{\circ}$ & $30^{\circ}$ & $45^{\circ}$ \\
\hline Dom & $1.5 \%$ & $0.9 \%$ & $-0.9 \%$ & NA & $-6.3 \%$ & $-14.0 \%$ & $-18.8 \%$ \\
\hline NonDom & $1.4 \%$ & $0.3 \%$ & $-0.5 \%$ & NA & $-7.7 \%$ & $-11.8 \%$ & $-18.7 \%$ \\
\hline
\end{tabular}


1

Quantifying median nerve cross-sectional area (MNCSA), median nerve longitudinal diameter (D1), and median nerve vertical diameter (D2) at the proximal carpal tunnel.

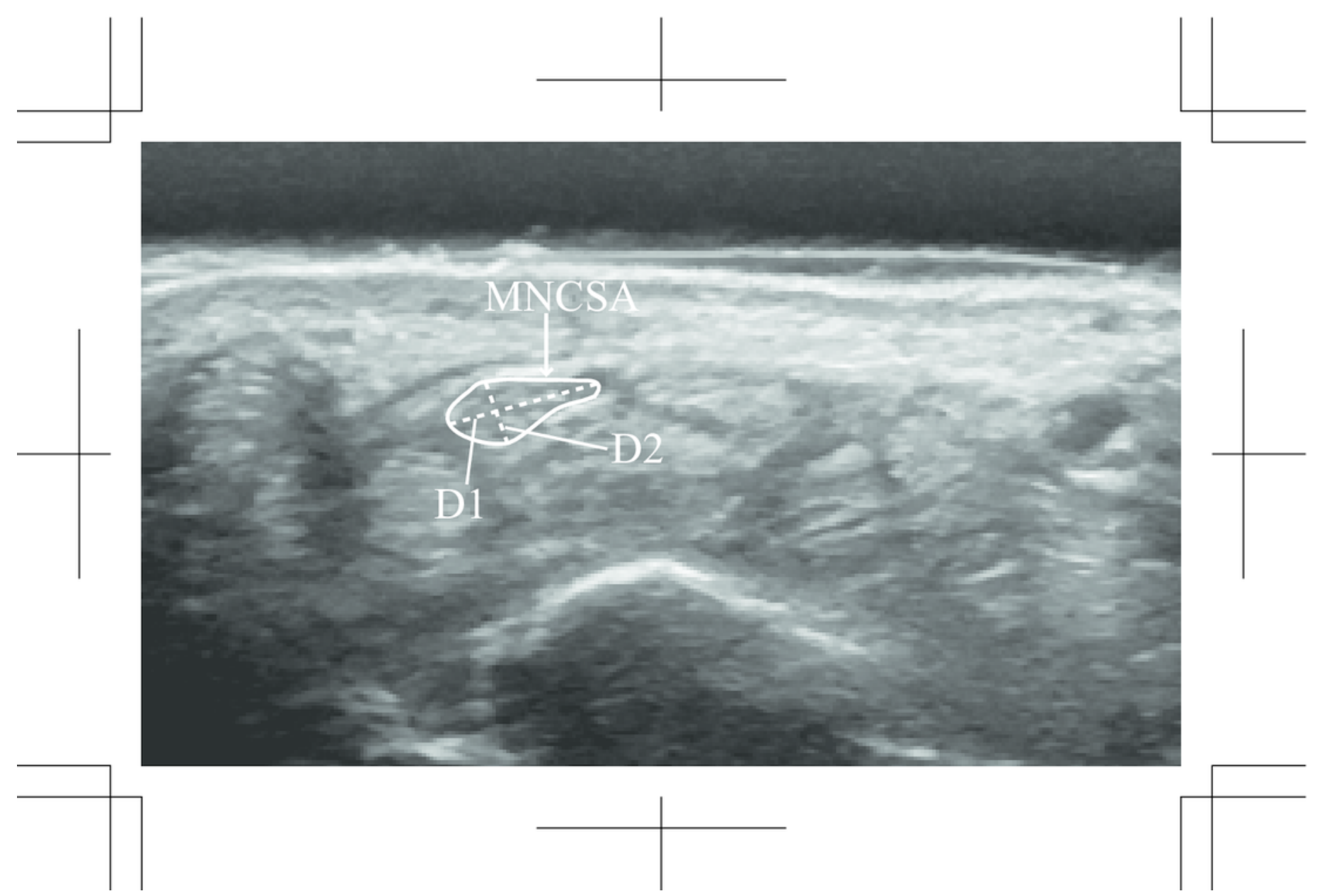


2

Deformation percentages between young and older adults. (a) Longitudinal diameter, D1 (b) Vertical diameter, D2.

* Cited from Loh \& Muraki (2015) 

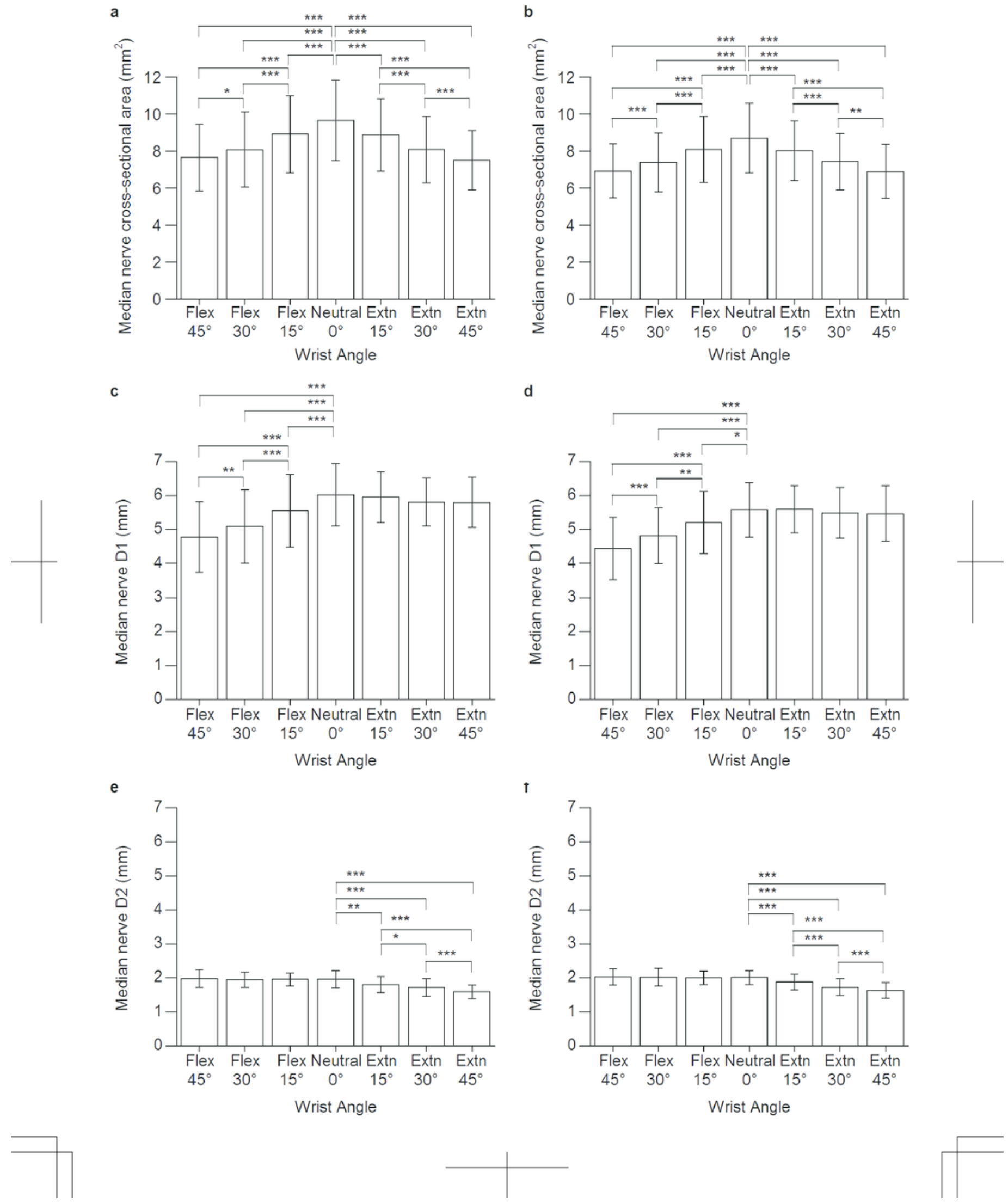


\section{3}

Median nerve cross-sectional area at different wrist angles.

(a) Dominant hand. (b) Nondominant hand; Median nerve longitudinal diameter (D1) at different wrist angles (c) Dominant hand. (d) Nondominant hand; Median nerve vertical diameter (D2) at different wrist angles (e) Dominant hand (f) Nondominant hand. Extn = extension, Flex $=$ flexion. $* \mathrm{P}<.05, * * \mathrm{P}<.01, * * * \mathrm{P}<.001$.
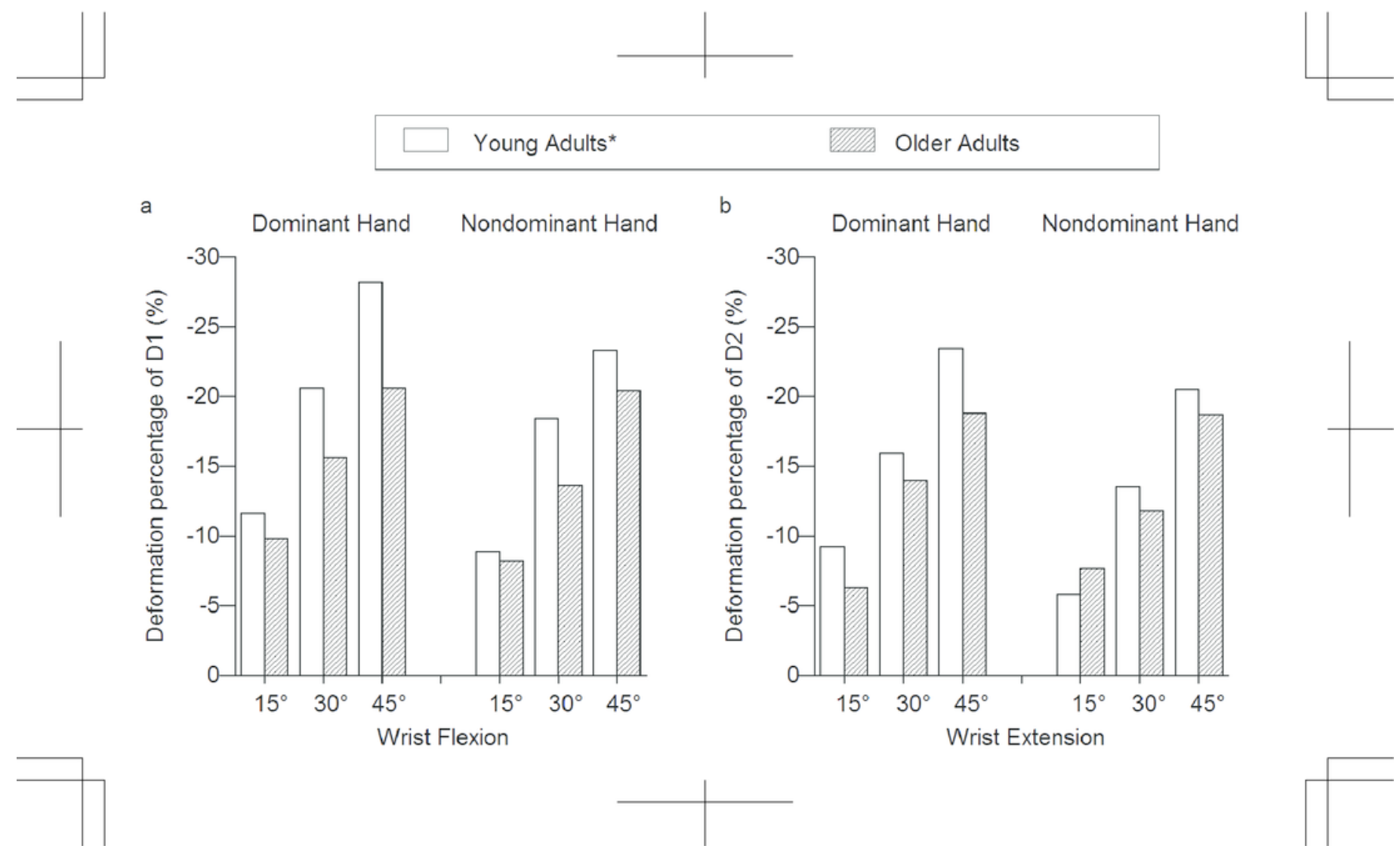